\title{
UNIQUENESS OF SOLUTIONS FOR FOURTH-ORDER NONLOCAL BOUNDARY VALUE PROBLEMS
}

\author{
JOHNNY HENDERSON AND DING MA
}

Received 19 January 2006; Accepted 22 January 2006

Uniqueness implies uniqueness relationships are examined among solutions of the fourth-order ordinary differential equation, $y^{(4)}=f\left(x, y, y^{\prime}, y^{\prime \prime}, y^{\prime \prime \prime}\right)$, satisfying 5-point, 4-point, and 3-point nonlocal boundary conditions.

Copyright (c) 2006 J. Henderson and D. Ma. This is an open access article distributed under the Creative Commons Attribution License, which permits unrestricted use, distribution, and reproduction in any medium, provided the original work is properly cited.

\section{Introduction}

We are concerned with uniqueness of solutions of certain nonlocal boundary value problems for the fourth-order ordinary differential equation,

$$
y^{(4)}=f\left(x, y, y^{\prime}, y^{\prime \prime}, y^{\prime \prime \prime}\right), \quad a<x<b
$$

where

(A) $f:(a, b) \times \mathbb{R}^{4} \rightarrow \mathbb{R}$ is continuous,

(B) solutions of initial value problems for (1.1) are unique and exist on all of $(a, b)$. By uniqueness of solutions, our meaning is uniqueness of solutions, when solutions exist.

In particular, we deal with "uniqueness implies uniqueness" relationships among solutions of (1.1) satisfying nonlocal 5-point boundary conditions,

$$
\begin{gathered}
y\left(x_{1}\right)=y_{1}, \quad y\left(x_{2}\right)=y_{2}, \\
y\left(x_{3}\right)=y_{3}, \quad y\left(x_{4}\right)-y\left(x_{5}\right)=y_{4}, \\
y\left(x_{1}\right)-y\left(x_{2}\right)=y_{1}, \quad y\left(x_{3}\right)=y_{2}, \\
y\left(x_{4}\right)=y_{3}, \quad y\left(x_{5}\right)=y_{4},
\end{gathered}
$$

where $a<x_{1}<x_{2}<x_{3}<x_{4}<x_{5}<b$, with solutions of (1.1) satisfying nonlocal 4-point 
boundary conditions given by

$$
\begin{aligned}
& y\left(x_{1}\right)=y_{1}, \quad y^{\prime}\left(x_{1}\right)=y_{2}, \quad y\left(x_{2}\right)=y_{3}, \quad y\left(x_{3}\right)-y\left(x_{4}\right)=y_{4}, \\
& y\left(x_{1}\right)-y\left(x_{2}\right)=y_{1}, \quad y\left(x_{3}\right)=y_{2}, \quad y\left(x_{4}\right)=y_{3}, \quad y^{\prime}\left(x_{4}\right)=y_{4}, \\
& y\left(x_{1}\right)=y_{1}, \quad y\left(x_{2}\right)=y_{2}, \quad y^{\prime}\left(x_{2}\right)=y_{3}, \quad y\left(x_{3}\right)-y\left(x_{4}\right)=y_{4}, \\
& y\left(x_{1}\right)-y\left(x_{2}\right)=y_{1}, \quad y\left(x_{3}\right)=y_{2}, \quad y^{\prime}\left(x_{3}\right)=y_{3}, \quad y\left(x_{4}\right)=y_{4},
\end{aligned}
$$

where $a<x_{1}<x_{2}<x_{3}<x_{4}<b$, as well as with solutions of (1.1) satisfying nonlocal 3point boundary conditions given by

$$
\begin{aligned}
& y\left(x_{1}\right)=y_{1}, \quad y^{\prime}\left(x_{1}\right)=y_{2}, \quad y^{\prime \prime}\left(x_{1}\right)=y_{3}, \quad y\left(x_{2}\right)-y\left(x_{3}\right)=y_{4}, \\
& y\left(x_{1}\right)-y\left(x_{2}\right)=y_{1}, \quad y\left(x_{3}\right)=y_{2}, \quad y^{\prime}\left(x_{3}\right)=y_{3}, \quad y^{\prime \prime}\left(x_{3}\right)=y_{4},
\end{aligned}
$$

where $a<x_{1}<x_{2}<x_{3}<b$, and in each case $y_{1}, y_{2}, y_{3}, y_{4} \in \mathbb{R}$.

Questions involving "uniqueness implies uniqueness" for solutions of boundary value problems for ordinary differential equations enjoy some history. Jackson's monumental works $[20,21]$ dealt with this question for solutions of $k$-point conjugate boundary value problems for $n$ th-order ordinary differential equations. Later, Henderson [12] dealt with this question for $k$-point right focal boundary value problems for $n$ th-order ordinary differential equations. Other uniqueness implies uniqueness results are found in the papers by Clark and Henderson [2], Ehme and Hankerson [4], Henderson and McGwier [17], and Peterson [39].

The questions in this paper involve (i) whether uniqueness of solutions of (1.1), (1.2) implies uniqueness of solutions of (1.1), (1.j), $j=4,6,8$, and (ii) whether uniqueness of solutions of (1.1), (1.j), $j=4, \ldots, 9$, imply uniqueness of solutions (1.1), (1.2) and (1.1), (1.3). A principal reason for considering questions such as (i) or (ii) is that such results often imply the existence of solutions for boundary value problems; see for example $[1,9$ $11,13-15,17,18,22,24,26,27]$.

The literature is vast on fourth-order nonlinear boundary value problems, and we cite $[3,5,23,28-30,33,35,36,38,40]$ as a list for just a few of these papers dealing with both theoretical issues as well as application models. In addition, nonlocal boundary value problems have received a good deal of research attention. For a brief overview of some research devoted to nonlocal boundary value problems, we suggest the list of papers [6$8,16,19,25,31,32,34,37,43,44]$.

The motivation for this paper is two-fold. First, it would be the work by Peterson [39] in which he showed that, for the fourth-order equation (1.1), uniqueness of solutions of 4-point "conjugate" boundary value problems is equivalent to uniqueness of both 2point and 3-point "conjugate" boundary value problems. Second, it would be a recent paper by Clark and Henderson [2] in which they established for "third-order" differential equations, uniqueness of solutions of 4-point nonlocal boundary value problems is equivalent to uniqueness of solutions of both 2-point and 3-point nonlocal boundary value problems. 


\section{Uniqueness results for conjugate problems}

In this section, we will state some of the motivational uniqueness results due to Peterson [39] for conjugate boundary value problems for (1.1). In particular, Peterson dealt with relationships among boundary value problems for (1.1) satisfying 4-point conjugate boundary conditions of the form

$$
y\left(x_{1}\right)=y_{1}, \quad y\left(x_{2}\right)=y_{2}, \quad y\left(x_{3}\right)=y_{3}, \quad y\left(x_{4}\right)=y_{4},
$$

$a<x_{1}<x_{2}<x_{3}<x_{4}<b$, along with solutions of (1.1) satisfying 3-point conjugate boundary value problems of the form

$$
\begin{array}{llll}
y\left(x_{1}\right)=y_{1}, & y^{\prime}\left(x_{1}\right)=y_{2}, & y\left(x_{2}\right)=y_{3}, & y\left(x_{3}\right)=y_{4}, \\
y\left(x_{1}\right)=y_{1}, & y\left(x_{2}\right)=y_{2}, & y^{\prime}\left(x_{2}\right)=y_{3}, & y\left(x_{3}\right)=y_{4}, \\
y\left(x_{1}\right)=y_{1}, & y\left(x_{2}\right)=y_{2}, & y\left(x_{3}\right)=y_{3}, & y^{\prime}\left(x_{3}\right)=y_{4},
\end{array}
$$

$a<x_{1}<x_{2}<x_{3}<b$, as well as with solutions of (1.1) satisfying 2-point conjugate boundary value problems of the form

$$
\begin{array}{llll}
y\left(x_{1}\right)=y_{1}, & y^{\prime}\left(x_{1}\right)=y_{2}, & y^{\prime \prime}\left(x_{1}\right)=y_{3}, & y\left(x_{2}\right)=y_{4}, \\
y\left(x_{1}\right)=y_{1}, & y^{\prime}\left(x_{1}\right)=y_{2}, & y\left(x_{2}\right)=y_{3}, & y^{\prime}\left(x_{2}\right)=y_{4}, \\
y\left(x_{1}\right)=y_{1}, & y\left(x_{2}\right)=y_{2}, & y^{\prime}\left(x_{2}\right)=y_{3}, & y^{\prime \prime}\left(x_{2}\right)=y_{4},
\end{array}
$$

$a<x_{1}<x_{2}<b$, and in each case $y_{1}, y_{2}, y_{3}, y_{4} \in \mathbb{R}$.

A major part of Peterson's work dealt with establishing the next result.

Theorem 2.1. Assume conditions $(A)$ and $(B)$ are satisfied. Let $k_{0} \in\{2,3,4\}$ be given, and assume that solutions of $k_{0}$-point conjugate boundary value problems for (1.1) are unique on $(a, b)$. Then, for each $k \in\{2,3,4\} \backslash\left\{k_{0}\right\}$, solutions of $k$-point conjugate boundary value problems for (1.1) are unique on $(a, b)$.

It follows, in turn, from a "uniqueness implies existence" result of Hartman [10] and Klaasen [24] for conjugate boundary value problems that, under the hypotheses of Theorem 2.1, solutions of conjugate boundary value problems for (1.1) actually exist.

Theorem 2.2. Assume the hypotheses of Theorem 2.1. Then for $k \in\{2,3,4\}$, each $k$-point conjugate boundary value problem for (1.1) has a unique solution on $(a, b)$.

\section{Uniqueness of 5-point implies uniqueness of 4-point and 3-point}

In this section, we show that uniqueness of solutions of 5-point nonlocal boundary value problems for (1.1) implies uniqueness of solutions for both 4-point and 3-point nonlocal boundary value problems. In addition to hypotheses (A) and (B), we will draw upon some uniqueness conditions for the 5-point nonlocal problems (1.1), (1.2) and (1.1), (1.3). 
4 Fourth-order nonlocal boundary value problems

(C) Given $a<x_{1}<x_{2}<x_{3}<x_{4}<x_{5}<b$, if $y(x)$ and $z(x)$ are two solutions of (1.1) satisfying

$$
\begin{gathered}
y\left(x_{1}\right)=z\left(x_{1}\right), \quad y\left(x_{2}\right)=z\left(x_{2}\right), \quad y\left(x_{3}\right)=z\left(x_{3}\right), \\
y\left(x_{4}\right)-y\left(x_{5}\right)=z\left(x_{4}\right)-z\left(x_{5}\right)
\end{gathered}
$$

then $y(x)=z(x), a<x<b$.

(D) Given $a<x_{1}<x_{2}<x_{3}<x_{4}<x_{5}<b$, if $y(x)$ and $z(x)$ are two solutions of (1.1) satisfying

$$
\begin{gathered}
y\left(x_{1}\right)-y\left(x_{2}\right)=z\left(x_{1}\right)-z\left(x_{2}\right), \quad y\left(x_{3}\right)=z\left(x_{3}\right), \\
y\left(x_{4}\right)=z\left(x_{4}\right), \quad y\left(x_{5}\right)=z\left(x_{5}\right)
\end{gathered}
$$

then $y(x)=z(x), a<x<b$.

Remarks 3.1. (a) We note that, under either assumption (C) or (D), solutions of 4-point "conjugate" boundary value problems for (1.1) are unique, when they exist. That is, if $y(x)$ and $z(x)$ are both solutions of (1.1) such that, for some points $a<t_{1}<t_{2}<t_{3}<t_{4}<b$, $y\left(t_{i}\right)=z\left(t_{i}\right), i=1,2,3,4$, then by the intermediate value theorem, there exist $t_{1}<\tau_{1}<\tau_{2}<$ $t_{2}<t_{3}<\sigma_{1}<\sigma_{2}<t_{4}$ such that, both $y\left(\tau_{1}\right)-y\left(\tau_{2}\right)=z\left(\tau_{1}\right)-z\left(\tau_{2}\right), y\left(t_{i}\right)=z\left(t_{i}\right), i=2,3,4$, and $y\left(t_{i}\right)=z\left(t_{i}\right), i=1,2,3, y\left(\sigma_{1}\right)-y\left(\sigma_{2}\right)=z\left(\sigma_{1}\right)-z\left(\sigma_{2}\right)$. Namely, if either (C) or (D) holds, then $y(x)=z(x)$.

(b) As a consequence, if either (A), (B), and (C), or (A), (B), and (D) are assumed, then Theorem 2.2 implies that each $k$-point "conjugate" boundary value problem for (1.1), $k=2,3,4$, has a unique solution.

Behind the uniqueness results of this section is the role of continuous dependence of solutions on boundary conditions. This continuous dependence arises somewhat from applications of the Brouwer theorem on invariance of domain [41] in conjunction with continuous dependence of solutions on initial conditions. We present our first such continuous dependence result. The proof is rather standard in the context of uniqueness properties on solutions with respect to both initial conditions and boundary conditions. So we will omit the details of the proof, but we suggest $[2,21]$ as good references for typical arguments used in the proof.

Theorem 3.2. Assume (A), (B), and (C), and let $z(x)$ be an arbitrary solution of (1.1). Then, for any $a<x_{1}<x_{2}<x_{3}<x_{4}<x_{5}<b$ and $a<c<x_{1}$, and $x_{5}<d<b$, and given any $\epsilon>0$, there exists $\delta(\epsilon,[c, d])>0$, so that $\left|x_{i}-t_{i}\right|<\delta, 1 \leq i \leq 5,\left|z\left(x_{i}\right)-y_{i}\right|<\delta, i=1,2,3$, and $\left|z\left(x_{4}\right)-z\left(x_{5}\right)-y_{4}\right|<\delta$ imply that (1.1) has a solution $y(x)$ with

$$
\begin{gathered}
y\left(t_{i}\right)=y_{i}, \quad i=1,2,3, \\
y\left(t_{4}\right)-y\left(t_{5}\right)=y_{4},
\end{gathered}
$$

and $\left|y^{(i-1)}(x)-z^{(i-1)}(x)\right|<\epsilon$ on $[c, d], i=1,2,3,4$.

We now proceed to establish a sequence of theorems exhibiting that uniqueness of solutions of (1.1), (1.2) implies uniqueness of solutions of (1.1), (1.j), $j=4,6,8$. 
Theorem 3.3. Assume (A), (B), and (C) are satisfied. Then solutions of (1.1), (1.4) are unique when they exist.

Proof. Suppose (1.1), (1.4) has two solutions $y(x)$ and $z(x)$, and let us say

$$
\begin{gathered}
z\left(x_{1}\right)=y\left(x_{1}\right), \quad z^{\prime}\left(x_{1}\right)=y^{\prime}\left(x_{1}\right), \\
z\left(x_{2}\right)=y\left(x_{2}\right), \quad z\left(x_{3}\right)-z\left(x_{4}\right)=y\left(x_{3}\right)-y\left(x_{4}\right),
\end{gathered}
$$

for some $a<x_{1}<x_{2}<x_{3}<x_{4}<b$. By uniqueness of 2-point conjugate boundary value problems for (1.1), $z^{\prime \prime}\left(x_{1}\right) \neq y^{\prime \prime}\left(x_{1}\right)$ and $z^{\prime}\left(x_{2}\right) \neq y^{\prime}\left(x_{2}\right)$.

Without loss of generality, we assume $y(x)>z(x)$ on $\left(a, x_{2}\right) \backslash\left\{x_{1}\right\}$. Then $y(x)<z(x)$ on $\left(x_{2}, b\right)$. Fix $a<\tau<x_{1}$. By Theorem 3.2, for $\epsilon>0$ sufficiently small, there exist a $\delta>0$ and a solution $z_{\delta}(x)$ of (1.1) satisfying

$$
\begin{gathered}
z_{\delta}(\tau)=z(\tau), \quad z_{\delta}\left(x_{1}\right)=z\left(x_{1}\right)+\delta, \\
z_{\delta}\left(x_{2}\right)=z\left(x_{2}\right)=y\left(x_{2}\right), \\
z_{\delta}\left(x_{3}\right)-z_{\delta}\left(x_{4}\right)=z\left(x_{3}\right)-z\left(x_{4}\right)=y\left(x_{3}\right)-y\left(x_{4}\right),
\end{gathered}
$$

and $\left|z_{\delta}^{(i-1)}(x)-z^{(i-1)}(x)\right|<\epsilon, i=1,2,3,4$, on $\left[\tau, x_{4}\right]$. For $\epsilon$ small, there exists $\tau<\sigma_{1}<x_{1}<$ $\sigma_{2}<x_{2}$ so that

$$
\begin{gathered}
z_{\delta}\left(\sigma_{1}\right)=y\left(\sigma_{1}\right), \quad z_{\delta}\left(\sigma_{2}\right)=y\left(\sigma_{2}\right) \\
z_{\delta}\left(x_{2}\right)=y\left(x_{2}\right), \quad z_{\delta}\left(x_{3}\right)-z_{\delta}\left(x_{4}\right)=y\left(x_{3}\right)-y\left(x_{4}\right) .
\end{gathered}
$$

By assumption $(\mathrm{C}), z_{\delta}(x)=y(x)$ on $(a, b)$. However, $z_{\delta}\left(x_{1}\right)=z\left(x_{1}\right)+\delta=y\left(x_{1}\right)+\delta>$ $y\left(x_{1}\right)$, which is a contradiction.

So solutions of (1.1), (1.4) are unique.

Remark 3.4. In view of Theorem 3.3, we remark that, as in Theorem 3.2, solutions of the nonlocal problem (1.1), (1.4) depend continuously on 4-point nonlocal boundary conditions. This type of remark will hold true following each of the subsequent uniqueness results.

Theorem 3.5. Assume (A), (B), and (C) are satisfied. Then solutions of (1.1), (1.6) are unique when they exist.

Proof. Suppose (1.1), (1.6) has two solutions $y(x)$ and $z(x)$, and let us say

$$
\begin{gathered}
z\left(x_{1}\right)=y\left(x_{1}\right), \quad z\left(x_{2}\right)=y\left(x_{2}\right), \\
z^{\prime}\left(x_{2}\right)=y^{\prime}\left(x_{2}\right), \quad z\left(x_{3}\right)-z\left(x_{4}\right)=y\left(x_{3}\right)-y\left(x_{4}\right),
\end{gathered}
$$

for some $a<x_{1}<x_{2}<x_{3}<x_{4}<b$. By uniqueness of solutions of 2-point conjugate boundary value problems for $(1.1), z^{\prime}\left(x_{1}\right) \neq y^{\prime}\left(x_{1}\right)$ and $z^{\prime \prime}\left(x_{2}\right) \neq y^{\prime \prime}\left(x_{2}\right)$.

Without loss of generality, we assume $y(x)>z(x)$ on $\left(x_{1}, b\right) \backslash\left\{x_{2}\right\}$. Then $y(x)<z(x)$ on $\left(a, x_{1}\right)$. Fix $x_{1}<\tau<x_{2}$. By Theorem 3.2, for $\epsilon>0$ sufficiently small, there exists a $\delta>0$ 
6 Fourth-order nonlocal boundary value problems

and a solution $z_{\delta}(x)$ of (1.1) satisfying

$$
\begin{gathered}
z_{\delta}\left(x_{1}\right)=z\left(x_{1}\right)=y\left(x_{1}\right), \quad z_{\delta}(\tau)=z(\tau), \quad z_{\delta}\left(x_{2}\right)=z\left(x_{2}\right)+\delta \\
z_{\delta}\left(x_{3}\right)-z_{\delta}\left(x_{4}\right)=z\left(x_{3}\right)-z\left(x_{4}\right)=y\left(x_{3}\right)-y\left(x_{4}\right)
\end{gathered}
$$

and $\left|z_{\delta}^{(i-1)}(x)-z^{(i-1)}(x)\right|<\epsilon, i=1,2,3,4$, on $\left[\tau, x_{4}\right]$. For $\epsilon$ small, there exists $x_{1}<\sigma_{1}<$ $x_{2}<\sigma_{2}<x_{4}$ so that

$$
\begin{gathered}
z_{\delta}\left(x_{1}\right)=y\left(x_{1}\right), \quad z_{\delta}\left(\sigma_{1}\right)=y\left(\sigma_{1}\right), \\
z_{\delta}\left(\sigma_{2}\right)=y\left(\sigma_{2}\right), \quad z_{\delta}\left(x_{3}\right)-z_{\delta}\left(x_{4}\right)=y\left(x_{3}\right)-y\left(x_{4}\right) .
\end{gathered}
$$

By assumption $(\mathrm{C}), z_{\delta}(x)=y(x)$ on $(a, b)$. However, $z_{\delta}\left(x_{2}\right)=z\left(x_{2}\right)+\delta=y\left(x_{2}\right)+\delta>$ $y\left(x_{2}\right)$, which is a contradiction.

So solutions of (1.1), (1.6) are unique.

Theorem 3.6. Assume (A), (B), and (C) are satisfied. Then solutions of (1.1), (1.8) are unique when they exist.

Proof. Suppose (1.1), (1.8) has two solutions $y(x)$ and $z(x)$ satisfying

$$
\begin{gathered}
y\left(x_{1}\right)=z\left(x_{1}\right), \quad y^{\prime}\left(x_{1}\right)=z^{\prime}\left(x_{1}\right), \\
y^{\prime \prime}\left(x_{1}\right)=z^{\prime \prime}\left(x_{1}\right), \quad y\left(x_{2}\right)-y\left(x_{3}\right)=z\left(x_{2}\right)-z\left(x_{3}\right),
\end{gathered}
$$

for some $a<x_{1}<x_{2}<x_{3}<b$. Now $y^{\prime \prime \prime}\left(x_{1}\right) \neq z^{\prime \prime \prime}\left(x_{1}\right)$, and we may assume $y^{\prime \prime \prime}\left(x_{1}\right)>$ $z^{\prime \prime \prime}\left(x_{1}\right)$.

By the last remark above, solutions of (1.1), (1.4) depend continuously on their boundary conditions. Fix $x_{1}<\rho<x_{2}$. For $\epsilon>0$ small, there is a $\delta>0$ and a solution $z_{\delta}(x)$ satisfying

$$
\begin{gathered}
z_{\delta}\left(x_{1}\right)=z\left(x_{1}\right)=y\left(x_{1}\right), \quad z_{\delta}^{\prime}\left(x_{1}\right)=z^{\prime}\left(x_{1}\right)+\delta, \quad z_{\delta}(\rho)=z(\rho), \\
z_{\delta}\left(x_{2}\right)-z_{\delta}\left(x_{3}\right)=z\left(x_{2}\right)-z\left(x_{3}\right)=y\left(x_{2}\right)-y\left(x_{3}\right),
\end{gathered}
$$

and $\left|y^{(i-1)}(x)-z^{(i-1)}(x)\right|<\epsilon, i=1,2,3,4$, on $\left[x_{1}, x_{3}\right]$. For $\epsilon$ sufficiently small, there exist points $a<\tau_{1}<x_{1}<\tau_{2}<\rho$, which are in a neighborhood of $x_{1}$, such that $y(x)$ and $z_{\delta}(x)$ both satisfy

$$
\begin{gathered}
z_{\delta}\left(\tau_{1}\right)=y\left(\tau_{1}\right), \quad z_{\delta}\left(x_{1}\right)=y\left(x_{1}\right) \\
z_{\delta}\left(\tau_{2}\right)=y\left(\tau_{2}\right), \quad z_{\delta}\left(x_{2}\right)-z_{\delta}\left(x_{3}\right)=y\left(x_{2}\right)-y\left(x_{3}\right) .
\end{gathered}
$$

So we have $z_{\delta}(x)=y(x)$ on $(a, b)$ by hypothesis $(\mathrm{C})$. But

$$
z_{\delta}^{\prime}\left(x_{1}\right)=z^{\prime}\left(x_{1}\right)+\delta=y^{\prime}\left(x_{1}\right)+\delta>y^{\prime}\left(x_{1}\right) .
$$

This is a contradiction. So (1.1), (1.8) has at most one solution. 
Of course, in terms of the uniqueness condition (D), there are dual uniqueness results, which we now state as one theorem.

Theorem 3.7. Assume (A), (B), and (D) are satisfied. Then solutions of (1.1), (1.j), $j=$ $5,7,9$, are unique when they exist.

\section{Uniqueness of 4-point and 3-point implies uniqueness of 5-point}

In this section, our consideration is with a question converse to the uniqueness results of Section 3. In particular, we assume that solutions of 4-point and 3-point nonlocal boundary value problems for (1.1) are unique. It is then established that solutions of both (1.1), (1.2) and (1.1), (1.3) are also unique. Fundamental to our arguments is a Kamke type of convergence result for boundary value problems due to Vidossich [42], as well as a precompactness condition on bounded sequences of solutions of (1.1) due to Jackson and Schrader; see Agarwal [1]. We state both of those results at the outset of the section.

Theorem 4.1 (Vidossich). For each $n>0$, let $g_{n}:[c, d] \times \mathbb{R}^{N} \rightarrow \mathbb{R}$ be continuous, let $L_{n}$ : $C\left([c, d] \times \mathbb{R}^{N}, \mathbb{R}\right) \rightarrow \mathbb{R}^{N}$ be continuous, and let $r_{n} \in \mathbb{R}^{N}$. Assume that

(a) $\lim _{n} r_{n}=r_{0}$

(b) $\lim _{n} g_{n}=g_{0}$ and $\lim _{n} L_{n}=L_{0}$ uniformly on compact subsets of $[c, d] \times \mathbb{R}^{N}$, respectively,

(c) each initial value problem,

$$
x^{\prime}=g_{n}(t, x), \quad x(a)=u,
$$

has at most one local solution for $u \in \mathbb{R}^{N}$,

(d) the functional boundary value problem,

$$
x^{\prime}=g_{0}(t, x), \quad L_{0}(x)=r,
$$

has at most one solution for each $r \in \mathbb{R}^{N}$.

Let $x_{0}$ be the solution to $x^{\prime}=g_{0}(t, x), L_{0}(x)=r_{0}$. Then for each $\epsilon>0$, there exists $n_{\epsilon}$ such that the functional boundary value problem,

$$
x^{\prime}=g_{n}(t, x), \quad L_{n}(x)=r_{n},
$$

has a solution $x_{n}$, for $n>n_{\epsilon}$, satisfying the condition

$$
\left\|x_{0}-x_{n}\right\|_{\infty}<\epsilon
$$

Theorem 4.2 (Jackson-Schrader). Assume that, with respect to (1.1), conditions (A) and (B) hold. In addition, assume that solutions of 4-point conjugate boundary value problems are unique. If $\left\{y_{k}(x)\right\}$ is a sequence of solutions of (1.1) for which there exists an interval $[c, d] \subset(a, b)$ and there exists an $M>0$ such that $\left|y_{k}(x)\right|<M$, for all $x \in[c, d]$ and for all $k \in \mathbb{N}$, then there exists a subsequence $\left\{y_{k_{j}}(x)\right\}$ such that, for $i=0,1,2,3,\left\{y_{k_{j}}^{(i)}(x)\right\}$ converges uniformly on each compact subinterval of $(a, b)$.

Remark 4.3. We remark that if solutions of (1.1) satisfying each of the nonlocal boundary conditions (1.j), $j=4, \ldots, 9$, are unique, when they exist, then solutions of 2 -point and 
8 Fourth-order nonlocal boundary value problems

3-point conjugate boundary value problems for (1.1) are unique. As a consequence of Theorems 2.1 and 2.2, it would follow that if (A) and (B) are also assumed, then each $k$-point conjugate boundary value problem for (1.1) has a solution which is unique, $k=$ $2,3,4$.

We now provide a type of converse to the results of Section 3.

Theorem 4.4. Assume (A) and (B) are satisfied. Assume solutions of (1.1) satisfying any of (1.j), $j=4, \ldots, 9$ are unique when they exist. Then solutions of both (1.1), (1.2) and (1.1), (1.3) are unique when they exist.

Proof. We establish the result for only (1.1), (1.2). Suppose (1.1), (1.2) has two distinct solutions $y(x)$ and $z(x)$, for some $a<x_{1}<x_{2}<x_{3}<x_{4}<x_{5}<b$ and some $y_{1}, y_{2}, y_{3}, y_{4} \in$ $\mathbb{R}$. That is,

$$
\begin{gathered}
y\left(x_{i}\right)=z\left(x_{i}\right), \quad i=1,2,3, \\
y\left(x_{4}\right)-y\left(x_{5}\right)=z\left(x_{4}\right)-z\left(x_{5}\right) .
\end{gathered}
$$

By assumptions (A) and (B) and uniqueness of solutions of 4-point and 3-point nonlocal boundary value problems, we know from the remark preceding the proof of this theorem that solutions of all conjugate boundary problems for (1.1) exist and are unique.

For each $n \geq 1$, let $y_{n}(x)$ be the solution of the boundary value problems for (1.1) satisfying the 3-point conjugate boundary conditions:

$$
\begin{gathered}
y_{n}\left(x_{3}\right)=y\left(x_{3}\right)=z\left(x_{3}\right), \quad y_{n}^{\prime}\left(x_{3}\right)=y^{\prime}\left(x_{3}\right)-n, \\
y_{n}\left(x_{4}\right)=y\left(x_{4}\right), \quad y_{n}\left(x_{5}\right)=y\left(x_{5}\right) .
\end{gathered}
$$

It follows from uniqueness of solutions of 4-point conjugate problems that, for $n \geq 1$,

$$
y(x)<y_{n}(x)<y_{n+1}(x)
$$

on $\left(a, x_{3}\right)$.

For each $n \geq 1$, let

$$
E_{n}=\left\{x: x_{1} \leq x \leq x_{2} \mid \text { where } y_{n}(x) \leq z(x)\right\} .
$$

We claim that $E_{n} \neq \varnothing$, for each $n \geq 1$. In that direction, suppose there exists $n_{0}$ so that $E_{n_{0}}=\varnothing$. Then $y_{n_{0}}(x)>z(x)$ on $\left[x_{1}, x_{2}\right]$.

Next, for all $\epsilon \geq 0$, let $y_{\epsilon}$ be the solution of (1.1) satisfying the 3-point conjugate boundary conditions:

$$
\begin{gathered}
y_{\epsilon}\left(x_{3}\right)=y\left(x_{3}\right)=z\left(x_{3}\right), \quad y_{\epsilon}^{\prime}\left(x_{3}\right)=y^{\prime}\left(x_{3}\right)-\epsilon, \\
y_{\epsilon}\left(x_{4}\right)=y\left(x_{4}\right), \quad y_{\epsilon}\left(x_{5}\right)=y\left(x_{5}\right) .
\end{gathered}
$$

Note when $\epsilon=0, y_{\epsilon}(x)=y(x)$. 
Define

$$
S=\left\{\epsilon \geq 0 \mid \text { for some } x_{1} \leq x \leq x_{2}, y_{\epsilon}(x) \leq z(x)\right\}
$$

$S \neq \varnothing$ since $0 \in S$. Now since $E_{n_{0}}=\varnothing, S$ is bounded above.

Let $\epsilon_{0}=\sup S$, and consider the solution $y_{\epsilon_{0}}(x)$ of (1.1). We claim that there exists $\tau \in$ $\left(x_{1}, x_{2}\right)$ so that $y_{\epsilon_{0}}(\tau) \leq z(\tau)$. If not, then $y_{\epsilon_{0}}(x)>z(x)$, for all $x_{1} \leq x \leq x_{2}$. By continuous dependence of solutions of (1.1) on 3-point conjugate boundary conditions, there exists $0<\epsilon_{1}<\epsilon_{0}$, so that $y_{\epsilon_{1}}(x)>z(x)$ for all $x_{1} \leq x \leq x_{2}$. Therefore $\epsilon_{1}$ is an upper bound of $S$. But by assumption $\epsilon_{0}=\sup S$, whereas $0<\epsilon_{1}<\epsilon_{0}$. This is a contradiction. Therefore there exists $\tau \in\left(x_{1}, x_{2}\right)$ so that $y_{\epsilon_{0}}(\tau) \leq z(\tau)$.

Next, if $y_{\epsilon_{0}}(\tau)<z(\tau)$, then by continuity, there exists an interval $[\tau-\rho, \tau+\rho]$ so that $y_{\epsilon_{0}}(x)<z(x)$ on $[\tau-\rho, \tau+\rho]$. So there exists $\epsilon_{0}<\epsilon_{2}$ so that $y_{\epsilon_{2}}(x) \leq z(x)$ on some interval $[\tau-\eta, \tau+\eta] \subset[\tau-\rho, \tau+\rho] \subset\left[x_{1}, x_{2}\right]$. So $\epsilon_{2} \in S$. But $\epsilon_{2}>\epsilon_{0}$, and so we contradict that $\epsilon_{0}$ is the least upper bound of $S$.

Now for this $\tau \in\left(x_{1}, x_{2}\right), y_{\epsilon_{0}}(\tau)=z(\tau)$, and $y_{\epsilon_{0}}(x) \geq z(x)$ for all $x \in\left[x_{1}, x_{2}\right] \backslash\{\tau\}$.

In particular,

$$
\begin{gathered}
y_{\epsilon_{0}}(\tau)=z(\tau), \quad y_{\epsilon_{0}}^{\prime}(\tau)=z^{\prime}(\tau), \\
y_{\epsilon_{0}}\left(x_{3}\right)=z\left(x_{3}\right), \quad y_{\epsilon_{0}}\left(x_{4}\right)-y_{\epsilon_{0}}\left(x_{5}\right)=z\left(x_{4}\right)-z\left(x_{5}\right) .
\end{gathered}
$$

By the uniqueness of solutions of 4-point nonlocal boundary value problems, we reach a contradiction. So $E_{n} \neq \varnothing$, for all $n \geq 1$.

Thus, $E_{n+1} \subset E_{n} \subset\left(x_{1}, x_{2}\right)$, for each $n \geq 1$, and each $E_{n}$ is also compact. Hence,

$$
\bigcap_{n=1}^{\infty} E_{n}:=E \neq \varnothing .
$$

Next, we observe that the set $E$ consists of a single point $\left\{x_{0}\right\}$ with $x_{1}<x_{0}<x_{2}$. To see this, suppose there are points $t_{1}, t_{2} \in E$ with $x_{1}<t_{1}<t_{2}<x_{2}$.

We claim that the interval $\left[t_{1}, t_{2}\right] \subseteq E$. Suppose to the contrary that there exists $\tau \in$ $\left(t_{1}, t_{2}\right)$ such that $\tau \notin E$. Then, there exists an $N \in \mathbb{N}$ such that, for each $n \geq N, y_{n}(\tau)>$ $z(\tau)$. By continuity, there exists a $\lambda>0$ such that, for each $n \geq N$,

$$
z(x)<y_{n}(x)<y_{n+1}(x), \quad x \in[\tau-\lambda, \tau+\lambda]
$$

With the solution $y_{\epsilon}(x)$ of (1.1) as defined above, we define a new set:

$$
S^{\prime}=\left\{\epsilon \geq 0 \mid \text { for some } \tau-\lambda \leq x \leq \tau-\lambda, y_{\epsilon}(x) \leq z(x)\right\} .
$$

Again $0 \in S^{\prime}$, and so $S^{\prime} \neq \varnothing$. In this case $N$ is an upper bound of $S^{\prime}$. We reach the same contradiction as above in showing the foregoing sets $E_{n}$ are nonnull. We conclude that the interval $\left[t_{1}, t_{2}\right] \subseteq E$, and the claim is verified.

However, $\left[t_{1}, t_{2}\right] \subseteq E$ implies that the sequence $\left\{y_{n}(x)\right\}$ is uniformly bounded on $\left[t_{1}, t_{2}\right]$. It follows from Theorem 4.2 that there is a subsequence $\left\{y_{n_{j}}(x)\right\}$ such that for each 
$i=0,1,2,3,\left\{y_{n_{j}}^{(i)}(x)\right\}$ converges uniformly on each compact subinterval of $(a, b)$. However,

$$
\lim _{j \rightarrow \infty} y_{n_{j}}^{\prime}\left(x_{3}\right)=\lim _{j \rightarrow \infty} y^{\prime}\left(x_{3}\right)-n_{j}=-\infty
$$

this is a contraction.

Thus we conclude that

$$
E=\left\{x_{0}\right\}
$$

with $x_{1}<x_{0}<x_{2}$, and we also have

$$
\lim _{n \rightarrow \infty} y_{n}\left(x_{0}\right) \leq z\left(x_{0}\right)
$$

Now, let $y_{0}(x)$ be the solution of the 4-point conjugate boundary value problem for (1.1) satisfying

$$
\begin{gathered}
\lim _{n \rightarrow \infty} y_{n}\left(x_{0}\right)=y_{0}\left(x_{0}\right), \quad y_{0}\left(x_{3}\right)=y\left(x_{3}\right)=z\left(x_{3}\right), \\
y_{0}\left(x_{4}\right)=y\left(x_{4}\right), \quad y_{0}\left(x_{5}\right)=y\left(x_{5}\right) .
\end{gathered}
$$

By Theorem 4.1, $\left\{y_{n}^{(i)}(x)\right\}$ converges to $y_{0}^{(i)}(x), i=0,1,2,3$, on each compact subinterval of $(a, b)$.

So $y_{0}\left(x_{0}\right) \leq z\left(x_{0}\right)$, which we claim that it leads to contradictions. There are two cases to resolve. First, assume $y_{0}\left(x_{0}\right)=z\left(x_{0}\right)$. Then we have two solutions $y_{0}(x)$ and $z(x)$ of (1.1) satisfying

$$
\begin{gathered}
y_{0}\left(x_{0}\right)=z\left(x_{0}\right), \quad y_{0}^{\prime}\left(x_{0}\right)=z^{\prime}\left(x_{0}\right), \quad y_{0}\left(x_{3}\right)=z\left(x_{3}\right), \\
y_{0}\left(x_{4}\right)-y_{0}\left(x_{5}\right)=y\left(x_{4}\right)-y\left(x_{5}\right)=z\left(x_{4}\right)-z\left(x_{5}\right),
\end{gathered}
$$

and so by uniqueness of solutions 4-point nonlocal boundary value problems (1.1), (1.4), $y_{0}(x) \equiv z(x)$ on $(a, b)$. This is a contradiction. So $\lim _{n \rightarrow \infty} y_{n}\left(x_{0}\right) \neq z\left(x_{0}\right)$.

The remaining case is that $y_{0}\left(x_{0}\right)<z\left(x_{0}\right)$. In this case, by the continuity of $y_{0}(x)$, there exists $\delta>0$ with $\left[x_{0}-\delta, x_{0}+\delta\right] \subset\left(x_{1}, x_{2}\right)$ on which $y_{0}(x)<z(x)$. Since $\lim _{n} y(x)=y_{0}(x)$ uniformly on each compact subinterval of $(a, b)$, it follows that $\left[x_{0}-\delta, x_{0}+\delta\right] \subset E$. This is a contradiction.

From this final contradiction, we conclude that $y_{0}\left(x_{0}\right) \leq z\left(x_{0}\right)$ is impossible. This resolves all situations, and we conclude that solutions of (1.1), (1.2) are unique. Of course, completely symmetric arguments yield that solutions of (1.1), (1.3) are also unique.

As a final statement, we present a theorem summarizing the results of this paper.

Theorem 4.5. Assume conditions (A) and (B) are satisfied. Then solutions of both (1.1), (1.2) and (1.1), (1.3) are unique when they exist, if and only if solutions of (1.1) satisfying each of (1.j), $j=4, \ldots, 9$, are unique when they exist. 


\section{References}

[1] R. P. Agarwal, Compactness condition for boundary value problems, Equadiff (Brno) 9 (1997), $1-23$.

[2] S. Clark and J. Henderson, Uniqueness implies existence and uniqueness criterion for nonlocal boundary value problems for third order differential equations, to appear in Proceedings of the American Mathematical Society.

[3] M. Conti, S. Terracini, and G. Verzini, Infinitely many solutions to fourth order superlinear periodic problems, Transactions of the American Mathematical Society 356 (2004), no. 8, 3283-3300.

[4] J. Ehme and D. Hankerson, Existence of solutions for right focal boundary value problems, Nonlinear Analysis 18 (1992), no. 2, 191-197.

[5] D. Franco, D. O'Regan, and J. Perán, Fourth-order problems with nonlinear boundary conditions, Journal of Computational and Applied Mathematics 174 (2005), no. 2, 315-327.

[6] J. R. Graef, C. Qian, and B. Yang, A three point boundary value problem for nonlinear fourth order differential equations, Journal of Mathematical Analysis and Applications 287 (2003), no. 1, 217233.

[7] C. P. Gupta, A Dirichlet type multi-point boundary value problem for second order ordinary differential equations, Nonlinear Analysis 26 (1996), no. 5, 925-931.

[8] C. P. Gupta, S. K. Ntouyas, and P. Ch. Tsamatos, Solvability of an m-point boundary value problem for second order ordinary differential equations, Journal of Mathematical Analysis and Applications 189 (1995), no. 2, 575-584.

[9] P. Hartman, Unrestricted n-parameter families, Rendiconti del Circolo Matematico di Palermo. Serie II 7 (1958), 123-142.

[10] _ On N-parameter families and interpolation problems for nonlinear ordinary differential equations, Transactions of the American Mathematical Society 154 (1971), 201-226.

[11] J. Henderson, Existence of solutions of right focal point boundary value problems for ordinary differential equations, Nonlinear Analysis 5 (1981), no. 9, 989-1002.

[12] _ Uniqueness of solutions of right focal point boundary value problems for ordinary differential equations, Journal of Differential Equations 41 (1981), no. 2, 218-227.

[13] $\ldots$ Right $\left(m ; \cdots ; m_{1}\right)$ boundary value problems for third order differential equations, Journal of Mathematical and Physical Sciences 18 (1984), no. 4, 405-413.

[14] Existence theorems for boundary value problems for nth-order nonlinear difference equations, SIAM Journal on Mathematical Analysis 20 (1989), no. 2, 468-478.

[15] Uniqueness implies existence for three-point boundary value problems for second order differential equations, Applied Mathematics Letters 18 (2005), no. 8, 905-909.

[16] J. Henderson, B. Karna, and C. C. Tisdell, Existence of solutions for three-point boundary value problems for second order equations, Proceedings of the American Mathematical Society 133 (2005), no. 5, 1365-1369.

[17] J. Henderson and R. W. McGwier Jr., Uniqueness, existence, and optimality for fourth-order Lipschitz equations, Journal of Differential Equations 67 (1987), no. 3, 414-440.

[18] J. Henderson and W. K. C. Yin, Existence of solutions for fourth order boundary value problems on a time scale, Journal of Difference Equations and Applications 9 (2003), no. 1, 15-28.

[19] V. A. Ill'in and E. I. Moiseev, Nonlocal boundary value problem of the second kind for a SturmLiouville operator, Differential Equations 23 (1987), no. 8, 979-987.

[20] L. K. Jackson, Existence and uniqueness of solutions of boundary value problems for third order differential equations, Journal of Differential Equations 13 (1973), no. 3, 432-437.

[21] Uniqueness of solutions of boundary value problems for ordinary differential equations, SIAM Journal on Applied Mathematics 24 (1973), no. 4, 535-538.

[22] B__ Boundary value problems for Lipschitz equations, Differential Equations (S. Ahmed, M. Keener, and A. Lazer, eds.), Academic Press, New York, 1980, pp. 31-50. 
[23] B. Karna, Extremal points for the fourth order boundary value problems, Mathematical Sciences Research Journal 7 (2003), no. 10, 382-393.

[24] G. A. Klaasen, Existence theorems for boundary value problems for nth order ordinary differential equations, The Rocky Mountain Journal of Mathematics 3 (1973), 457-472.

[25] L. Kong and Q. Kong, Positive solutions of nonlinear m-point boundary value problems on a measure chain, Journal of Difference Equations and Applications 9 (2003), no. 6, 615-627.

[26] A. Lasota and M. Łuczyński, A note on the uniqueness of two point boundary value problems I, Zeszyty Naukowe Uniwersytetu Jagiellonskiego, Prace Matematyczne 12 (1968), 27-29.

[27] A. Lasota and Z. Opial, On the existence and uniqueness of solutions of a boundary value problem for an ordinary second-order differential equation, Colloquium Mathematicum 18 (1967), 1-5.

[28] Y. Liu and W. Ge, Existence theorems of positive solutions for fourth-order four point boundary value problems, Analysis and Applications (Singapore) 2 (2004), no. 1, 71-85.

[29] H. Lü, H. Yu, and Y. Liu, Positive solutions for singular boundary value problems of a coupled system of differential equations, Journal of Mathematical Analysis and Applications 302 (2005), no. 1, 14-29.

[30] R. Ma, Some multiplicity results for an elastic beam equation at resonance, Applied Mathematics and Mechanics 14 (1993), no. 2, 193-200.

[31] _ Positive solutions for second-order three-point boundary value problems, Applied Mathematics Letters 14 (2001), no. 1, 1-5.

[32] __ Existence of positive solutions for superlinear semipositone m-point boundary-value problems, Proceedings of the Edinburgh Mathematical Society. Series II 46 (2003), no. 2, 279-292.

[33] _ Multiple positive solutions for a semipositone fourth-order boundary value problem, Hiroshima Mathematical Journal 33 (2003), no. 2, 217-227.

[34] R. Ma and N. Castaneda, Existence of solutions of nonlinear m-point boundary-value problems, Journal of Mathematical Analysis and Applications 256 (2001), no. 2, 556-567.

[35] J. J. Ma and F. R. Zhang, Solvability of a class of fourth-order two-point boundary value problems, Heilongjiang Daxue Ziran Kexue Xuebao 18 (2001), no. 2, 19-22.

[36] T. R. Marchant, Higher-order interaction of solitary waves on shallow water, Studies in Applied Mathematics 109 (2002), no. 1, 1-17.

[37] P. K. Palamides, Multi point boundary-value problems at resonance for n-order differential equations: positive and monotone solutions, Electronic Journal of Differential Equations 2004 (2004), no. $25,1-14$.

[38] M. H. Pei and S. K. Chang, Existence and uniqueness theorems of solutions for a class fourth-order boundary value problems, Kyungpook Mathematical Journal 41 (2001), no. 2, 299-309.

[39] D. E. Peterson, Uniqueness, existence, and comparison theorems for ordinary differential equations, Doctoral dissertation, Nebraska, 1973.

[40] A. C. Peterson, Focal Green's functions for fourth-order differential equations, Journal of Mathematical Analysis and Applications 75 (1980), no. 2, 602-610.

[41] E. H. Spanier, Algebraic Topology, McGraw-Hill, New York, 1966.

[42] G. Vidossich, On the continuous dependence of solutions of boundary value problems for ordinary differential equations, Journal of Differential Equations 82 (1989), no. 1, 1-14.

[43] J. R. L. Webb, Positive solutions of some three point boundary value problems via fixed point index theory, Nonlinear Analysis 47 (2001), no. 7, 4319-4332.

[44] Z. Zhang and J. Wang, Positive solutions to a second order three-point boundary value problem, Journal of Mathematical Analysis and Applications 285 (2003), no. 1, 237-249.

Johnny Henderson: Department of Mathematics, Baylor University, Waco, TX 76798-7328, USA

E-mail address: johnny_henderson@baylor.edu

Ding Ma: Department of Mathematics, Baylor University, Waco, TX 76798-7328, USA

E-mail address: ding_ma@baylor.edu 